\title{
Aproximación diagnóstica a la amiloidosis cardiaca por transtiretina en un área no endémica. ¿Qué variables no invasivas orientan a su diagnóstico?
}

\author{
Diagnostic approach to transthyretin amyloidosis in a non-endemic area. Which non- \\ invasive variables could guide the diagnosis?
}

\author{
Ainara Lozano-Bahamonde*, Amaia Lambarri-Izaguirre, Vanessa Escolar-Pérez e Inma Lluis-Serret \\ Servicio de Cardiología, Hospital Universitario Basurto, Bilbao, España
}

Al editor:

Clásicamente se ha considerado la amiloidosis cardiaca como una afección rara, con un amplio espectro de síntomas que requiere un alto índice de sospecha. Sin embargo, los estudios han demostrado que la amiloidosis cardiaca por transtiretina (TTR) es más común de lo que previamente se creía ${ }^{1,2}$. Las características clínicas que se han asociado a la amiloidosis cardiaca por TTR son el sexo masculino, la edad avanzada, la hipertrofia concéntrica y la función ventricular izquierda preservada ${ }^{1}$.

Se realizó un análisis descriptivo retrospectivo de las gammagrafías solicitadas en nuestro centro para descartar amiloidosis cardiaca por TTR desde septiembre de 2016 hasta noviembre de 2019. En dicho periodo se realizaron 39 gammagrafías, con una tendencia al alza en los últimos meses. Los objetivos fueron evaluar las gammagrafías solicitadas y conocer el porcentaje de gammagrafías diagnósticas de amiloidosis por TTR, establecer qué características son más frecuentes en los pacientes con amiloidosis por TTR en nuestra población de referencia y analizar las características diferenciales de las distintas posibilidades diagnósticas.
Del total de las pruebas, 22 (56.4\% de la muestra) mostraron una captación de grado 2-3 de Perugini, diagnóstica de amiloidosis por TTR. De acuerdo con las recomendaciones de diagnóstico no invasivo de amiloidosis cardiaca por $\mathrm{TTR}^{3}$, se descartó la presencia de pico monoclonal. Únicamente se realizó estudio genético a 10 pacientes, en dos de los cuales se detectó una mutación patogénica (Val50Met y variante patogénica c.290C>A en heterocigosis); los ocho restantes no mostraron mutaciones en el estudio molecular del gen TTR.

Hubo 16 pacientes $(41.0 \%)$ que mostraron captación de grado 0 y un paciente $(2.6 \%)$ con captación de grado 1. Este último presentaba, además, un componente clonal de cadena ligera lambda y proteinuria de Bence-Jones, por lo que se realizó una biopsia cardiaca que confirmó la positividad del amiloide para cadenas ligeras lambda mediante inmunohistoquímica. El diagnóstico definitivo en los pacientes con captación de grado 0-1 fue cardiopatía hipertensiva en 11 (28.2\%), amiloidosis AL en $3(7.7 \%$, confirmado por biopsia de médula ósea o biopsia miocárdica), miocardiopatía hipertrófica sarcomérica en 2 (5.1\%) y enfermedad de Fabry en 1 (2.6\%). Dada la elevada prevalencia de amiloidosis TTR y de cardiopatía hipertensiva en los estudios realizados

\section{Correspondencia:}

*Ainara Lozano-Bahamonde

E-mail: ainara.lozanobahamonde@osakidetza.eus licencia CC BY-NC-ND (http://creativecommons.org/licenses/by-nc-nd/4.0/).

Fecha de recepción: 24-08-2020

Fecha de aceptación: 03-11-2020

DOI: 10.24875/RCCAR.M21000033
Disponible en internet: 10-06-2021 Rev Colomb Cardiol. 2021;28(2):197-199 www.rccardiologia.com 
Tabla 1. Comparación de las características clínicas, ecocardiográficas y electrocardiográficas de ambos grupos

\begin{tabular}{|c|c|c|c|}
\hline & TTR & Cardiopatía HTA & $\mathbf{p}$ \\
\hline $\begin{array}{l}\text { Características demográficas } \\
\text { Sexo varón } \\
\text { Edad, años }\end{array}$ & $\begin{array}{l}19(86.4 \%) \\
81.5 \pm 6.02\end{array}$ & $\begin{array}{c}5(45.5 \%) \\
81.1 \pm 9.64\end{array}$ & $\begin{array}{l}0.033 \\
0.874\end{array}$ \\
\hline $\begin{array}{l}\text { Factores de riesgo cardiovascular } \\
\text { Tabaquismo } \\
\text { HTA } \\
\text { DM } \\
\text { Dislipidemia }\end{array}$ & $\begin{array}{l}6(27.3 \%) \\
15(68.2 \%) \\
2(9.1 \%) \\
11(50 \%)\end{array}$ & $\begin{array}{l}3(27.3 \%) \\
9(81.8 \%) \\
4(36.4 \%) \\
6(54.5 \%)\end{array}$ & $\begin{array}{c}1 \\
0.681 \\
0.146 \\
0.805\end{array}$ \\
\hline $\begin{array}{l}\text { Características clínicas y tratamiento } \\
\text { Hospitalización previa por IC } \\
\text { Sintomático } \\
\text { Disnea } \\
\text { Síncope } \\
\text { Palpitaciones } \\
\text { Túnel del carpo } \\
\text { PAS consulta, mmHg } \\
\text { PAD consulta, mmHg } \\
\text { IECA/ARA-II } \\
\text { Betabloqueador } \\
\text { NT-proBNP } \\
\text { Tiempo hasta gammagrafía, meses } \\
\text { Muertes }\end{array}$ & $\begin{array}{c}17(77.3 \%) \\
22(100 \%) \\
22(100 \%) \\
4(18.2 \%) \\
0(0 \%) \\
2(9.1 \%) \\
118.5 \pm 25.8 \\
66 \pm 6.7 \\
6(27.3 \%) \\
12(54.5 \%) \\
8013 \pm 11291.36 \\
19.6 \pm 23.8 \\
7(31.8 \%)\end{array}$ & $\begin{array}{c}6(54.5 \%) \\
10(90.9 \%) \\
10(90.9 \%) \\
0(0 \%) \\
1(9.1 \%) \\
0(0 \%) \\
141.6 \pm 19.1 \\
75.3 \pm 12.3 \\
7(63.6 \%) \\
3(27.3 \%) \\
2203 \pm 2509.9 \\
13.7 \pm 19.8 \\
1(9.1 \%)\end{array}$ & $\begin{array}{c}0.24 \\
0.33 \\
0.33 \\
0.276 \\
0.33 \\
0.542 \\
0.014 \\
0.008 \\
0.065 \\
0.14 \\
0.02 \\
0.502 \\
0.218\end{array}$ \\
\hline $\begin{array}{l}\text { Características ecocardiográficas } \\
\text { FEVI } \\
\text { Grosor máximo ETT, mm } \\
\text { Hipertrofia concéntrica } \\
\text { Dilatación VI } \\
\text { Patrón diastólico anormal } \\
\text { E/é } \\
\text { Al (AP), mm } \\
\text { HTAP > } 50 \text { mmHg } \\
\text { Afectación VD }\end{array}$ & $\begin{array}{c}48.6 \pm 13 \% \\
19.1 \pm 3.2 \\
17(77.3 \%) \\
3(13.6 \%) \\
8(90.9 \%) \\
17 \pm 6.6 \\
22 \pm 9.1 \\
7(31.8 \%) \\
7(31.8 \%)\end{array}$ & $\begin{array}{c}62.5 \pm 5.3 \% \\
13.3 \pm 3.2 \\
7(63.6 \%) \\
0(0 \%) \\
4(88.9 \%) \\
7 \pm 5.7 \\
11 \pm 4.1 \\
3(27.3 \%) \\
1(9.1 \%)\end{array}$ & $\begin{array}{c}0.002 \\
<0.001 \\
0.366 \\
0.534 \\
0.217 \\
0.46 \\
0.117 \\
1 \\
0.218\end{array}$ \\
\hline $\begin{array}{l}\text { Características electrocardiográficas } \\
\text { Fibrilación/flutter auricular } \\
\text { Bajo voltaje }(<10 \mathrm{~mm} \text { precordiales) } \\
\text { Signos indirectos HVI } \\
\text { Bloqueo de rama } \\
\text { BAV completo } \\
\text { Marcapasos }\end{array}$ & $\begin{array}{c}16(72.7 \%) \\
8(36.4 \%) \\
5(22.7 \%) \\
13(59.1 \%) \\
4(18.2 \%) \\
4(18.2 \%)\end{array}$ & $\begin{array}{c}7(63.6 \%) \\
1(9.1 \%) \\
1(9.1 \%) \\
4(36.4 \%) \\
0(0 \%) \\
1(9.1 \%)\end{array}$ & $\begin{array}{l}0.696 \\
0.212 \\
0.734 \\
0.218 \\
0.276 \\
0.643\end{array}$ \\
\hline
\end{tabular}

Al: aurícula izquierda; AP: anteroposterior; ARA-II: antagonistas de los receptores de la angiotensina II; BAV: bloqueo auriculoventricular; DM: diabetes

mellitus; E/é: relación entre la onda E derivada del Doppler pulsado a nivel de la válvula mitral y su relación con el Doppler tisular obtenido de la pared libre del ventrículo izquierdo a nivel mitral; ETT: ecocardiograma transtorácico; FEVI: fracción de eyección del ventrículo izquierdo; HTA: hipertensión arterial; HTAP: hipertensión arterial pulmonar; HVI: hipertrofia ventricular derecha; IC: insuficiencia cardiaca; IECA: inhibidores de la enzima convertidora de angiotensina; NT-proBNP: propéptido natriurético cerebral N-terminal; PAD: presión arterial diastólica; PAS: presión arterial sistólica; TTR: transtiretina; VD: ventrículo derecho; VI: ventrículo izquierdo.

y la trascendencia derivada del diagnóstico diferencial de las dos enfermedades, se realizó una comparación de las características clínicas, ecocardiográficas y electrocardiográficas de ambos grupos (Tabla 1).

De acuerdo con los resultados de nuestro estudio, las variables que con mayor frecuencia se asociaron a amiloidosis por TTR fueron el sexo masculino, el valor elevado de propéptido natriurético cerebral $\mathrm{N}$-terminal (NT-proBNP), la menor presión arterial en la consulta, la menor tolerancia al tratamiento con inhibidores de la enzima convertidora de angiotensina (IECA) y con antagonistas de los receptores de la angiotensina II (ARA-II), el grosor máximo mayor y la fracción de eyección ventricular izquierda (FEVI) más deprimida.

Así mismo, y aunque estas diferencias no resultaron estadísticamente significativas, se encontró mayor asociación con amiloidosis por TTR en pacientes con hospitalización previa por insuficiencia cardiaca, episodios sincopales previos, antecedentes de fibrilación o flutter auricular, bloqueo completo de rama derecha o izquierda, bloqueo auriculoventricular completo, marcapasos y antecedentes de túnel del carpo. 


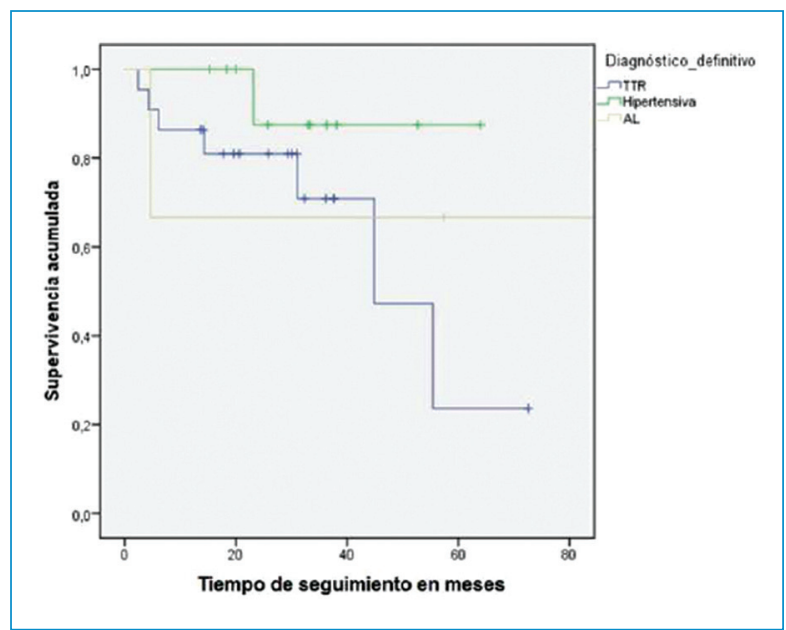

Figura 1. Curva de supervivencia acumulada según el diagnóstico definitivo de los pacientes.

Se destaca una mayor mortalidad (Fig. 1) de los pacientes con amiloidosis por TTR en comparación con los pacientes con cardiopatía hipertensiva, lo que subraya la importancia de un diagnóstico precoz, de cara a poder identificar aquellos que pudieran beneficiarse de un seguimiento más estrecho y, en casos seleccionados, recibir tratamientos dirigidos hacia la supresión, la estabilización o la degradación de la proteína TTR, pudiendo enlentecer la progresión y mejorar el pronóstico de estos pacientes.

Los resultados de este estudio orientan a que las gammagrafías se han realizado en pacientes con probabilidad pretest alta, lo que justifica que el porcentaje de pruebas con resultado positivo sea considerablemente mayor que el descrito en otras series (56.4 vs. $13.3 \%$ en la serie de González-López, et al. ${ }^{2}$ ). Además, respecto a otro estudio de González-López, et al. ${ }^{1}$, hay variables que orientan a que el diagnóstico se ha realizado en estadios más avanzados de la enfermedad, como son la edad avanzada (81.5 vs. 78.6 años), la clínica de insuficiencia cardiaca (100 vs. $67.6 \%$ ), la presión arterial sistólica más baja (118.5 vs. 126 $\mathrm{mm} \mathrm{Hg}$ ), la menor prescripción de IECA O ARA-II (27.3 vs. $41.7 \%$ ), un valor de NT-proBNP más alto
(8013 vs. 2997), una mayor incidencia de fibrilación o flutter auricular (72.7 vs. $55.6 \%$ ), una FEVI más deprimida (48.6 vs. $52 \%$ ) y un mayor espesor miocárdico (19.1 vs. $17.5 \mathrm{~mm}$ ).

Teniendo en cuenta nuestra serie podemos afirmar que el sexo masculino, un valor de NT-proBNP más alto, la presión arterial en la consulta más baja, una FEVI más deprimida y un grosor mayor en el ecocardiograma son variables que orientan a una probable amiloidosis por TTR. Además, se describen otras variables que son más frecuentes en el grupo de amiloidosis por TTR y que probablemente resultarían significativas si el tamaño de la muestra fuera mayor.

\section{Conflicto de intereses}

Los autores declaran que no existen conflictos de intereses.

\section{Responsabilidades éticas}

Protección de personas y animales. Los autores declaran que para esta investigación no se han realizado experimentos en seres humanos ni en animales.

Confidencialidad de los datos. Los autores declaran que han seguido los protocolos de su centro de trabajo sobre la publicación de datos de pacientes.

Derecho a la privacidad y consentimiento informado. Los autores declaran que en este artículo no aparecen datos de pacientes.

\section{Bibliografía}

1. González-López E, Gagliardi C, Domínguez F, Cándida Quarta C, de Haro-del Moral FJ, Milandri A, et al. Clinical characteristics of wild-type transthyretin cardiac amyloidosis: disproving myths. Eur Heart J. 2017;38:1895-904. Disponible en: https://doi.org/10.1093/eurheartj/ ehx043.

2. González-López E, Gallego-Delgado M, Guzzo-Merello G, de Haro-del Moral FJ, Cobo-Marcos M, Robles C, et al. Wild-type transthyretin amyloidosis as a cause of heart failure with preserved ejection fraction. Eur Heart J. 2015;36:2585ロ94. Disponible en: https://doi.org/10.1093/ eurheartj/ehv338.

3. Gillmore JD, Maurer MS, Falk RH, Merlini G, Damy T, Dispenzieri A, et al. Nonbiopsy diagnosis of cardiac transthyretin amyloidosis. Circulation. 2016;133:2404-12. Disponible en: https://doi.org/10.1161/CIRCULATIONAHA.116.021612. 